\title{
Suppression of LETM1 by siRNA inhibits cell proliferation and invasion of bladder cancer cells
}

\author{
BISHENG HUANG $^{1 *}$, JINGWEI ZHANG ${ }^{1 *}$, XIAOLU ZHANG $^{1 *}$, CHI HUANG ${ }^{1,2}$, GUANGHUI HU $^{1}$, \\ SAIYANG LI ${ }^{1}$, TIANCHENG XIE ${ }^{1}$, MENGNAN LIU ${ }^{1}$ and YUNFEI XU ${ }^{1,2}$ \\ ${ }^{1}$ Department of Urology, Shanghai Tenth People's Hospital, Tongji University School of Medicine, Shanghai 200072; \\ ${ }^{2}$ Department of Urology, Shanghai Tenth People's Hospital, Nanjing Medical University, Nanjing, Jiangsu 210029, P.R. China
}

Received January 23, 2017; Accepted August 4, 2017

DOI: $10.3892 /$ or.2017.5959

\begin{abstract}
The leucine zipper-EF-hand containing transmembrane protein 1 (LETM1) is highly expressed in many human malignancies and is correlated with poor prognosis. However, the function of LETM1 in bladder cancer still remains unknown. In the present study, we analyzed the expression levels of LETM1 in bladder cancer tissues and non-cancerous tissues as well as in four bladder cancer cell lines (T24, EJ, 5637 and J82) and a human bladder epithelial immortalized cell line SV-HUC-1. Small interfering RNA (siRNA) was employed to knockdown the expression of LETM1 in the T24 cells. The proliferation of T24 cells was significantly repressed as evaluated by CCK- 8 assays. Transwell migration and invasion assays indicated that knockdown of LETM1 suppressed cell migration and invasion significantly. Flow cytometric analysis revealed that cells had accumulated at the S-phase when the expression of LETM1 was suppressed. Moreover, we found that several oncogenic proteins in the $\mathrm{Wnt} / \beta$-catenin signaling pathway, namely $\beta$-catenin, cyclin D1 and c-Myc were significantly decreased by the LETM1 siRNA. Collectively, these results revealed that the knockdown of LETM1 exhibited tumor suppressive effects, possibly by controlling the downstream $\mathrm{Wnt} / \beta$-catenin signaling pathway.
\end{abstract}

\section{Introduction}

Bladder cancer is one of the most common types of cancer worldwide. In 2015, the estimated newly diagnosed cases of bladder cancer were 74,000 and the estimated cancer-related deaths caused by bladder cancer were 16,000 in the United States (1). Currently, there are $~ 75 \%$ of cases that present with

Correspondence to: Professor Yunfei Xu, Department of Urology, Shanghai Tenth People's Hospital, Tongji University School of Medicine, 301 Yanchang Road, Shanghai 200072, P.R. China

E-mail: xuyunfeibb@sina.com

*Contributed equally

Key words: bladder cancer, LETM1, siRNA, proliferation, invasion non-muscle-invasive bladder cancer and $25 \%$ of cases that present with muscle-invasive bladder cancer $(2,3)$. Although there has been some progress in the clinical treatment of bladder cancer in the past years, the 5-year survival rate for patients with bladder cancer remains only $50-60 \%(4,5)$. Thus, it is urgent to reveal the potential molecular mechanisms involved in the tumorigenesis of bladder cancer.

Leucine zipper-EF-hand containing transmembrane protein 1 (LETM1), which was first identified in human Wolf-Hirschhorn syndrome $(6,7)$, is a mitochondrial inner membrane protein which plays an important role in mitochondrial ATP production and biogenesis by decreasing the mitochondrial mass and expression of many mitochondrial proteins (8). However, previous studies revealed that the high expression levels of LETM1 have been correlated with many human malignancies. For example, Chen et al reported that LETM1 was highly expressed in head and neck squamous cell carcinoma and that a high expression of LETM1 predicts poor prognosis (9). Another study revealed that high LETM1 expression was positively correlated with late clinical stage, poor differentiation, lymph node metastasis, disease-free survival and 10-year overall survival rates in triple-negative breast cancer (10). However, the role of LETM1 in human bladder cancer have yet to be determined. In this study, we investigated the role of LETM1 in bladder cancer. Our results demonstrated that the expression of LETM1 was significantly increased in bladder cancer tissues and cell lines, and that knockdown of LETM1 markedly decreased the proliferation, migration and invasion of bladder cancer cells. Moreover, the suppression of LETM1 induced the accumulation of S-phase cells.

\section{Materials and methods}

Patient data. Bladder cancer tissues and their matched adjacent normal tissues were obtained from the Shanghai Tenth People's Hospital, Tongji University School of Medicine (Shanghai, China). The study was approved by the Shanghai Tenth People's Hospital Ethics Committee and written informed consents were obtained from all patients.

Cell lines and cultures. The human bladder cancer cell lines (T24, EJ, 5637 and J82) and the human bladder epithelial immortalized SV-HUC-1 cell line were obtained from type 
culture collection of the Chinese Academy of Sciences (Shanghai, China). The SV-HUC-1 cells were cultured in F12K medium (Sigma-Aldrich, St. Louis, MO, USA). The T24, EJ and 5637 cells were cultured in RPMI-1640 medium (Gibco, Rockville, MD, USA) and the J82 cells were maintained in Dulbecco's modified Eagle's medium (DMEM; Gibco). These media were supplemented with $10 \%$ fetal bovine serum (FBS; Gibco) and $1 \%$ penicillin/streptomycin (HyClone, Logan, UT, USA). The cells were incubated at $37^{\circ} \mathrm{C}$ in a humidified atmosphere with $5 \% \mathrm{CO}_{2}$.

RNA isolation and quantitative real-time PCR. Total RNA was extracted from the cultured cells using TRIzol reagent (Invitrogen, Carlsbad, CA, USA) according to the manufacturer's instructions. The concentration and purity of the RNA were determined using an ND-2000 spectrophotometer (Thermo Fisher Scientific, Inc., Carlsbad, CA, USA). For the detection of the LETM1 mRNA level, the cDNA was synthesized using a PrimeScript RT Reagent kit (Takara Bio, Inc., Shiga, Japan) according to the manufacturer's instructions. qRT-PCR was performed with the KAPA SYBR FAST qPCR kit (Kapa Biosystems, Inc., Woburn, MA, USA). The primers for the qRT-PCR analysis were as follows: LETM1 sense, 5'-CTCAAGGAGGAGAGGCTGAA-3' and antisense, 5'-GAAGTTGTTGGTGCCGATG-3'; $\beta$-actin sense, 5'-CCTGGCACCCAGCACAAT-3' and antisense, 5'-GGGCCGGACTCGTCATAC-3'. The LETM1 mRNA level was normalized to the $\beta$-actin mRNA level. The data were analyzed using the $2^{-\Delta \Delta \mathrm{Ct}}$ method.

Immunohistochemical (IHC) analysis. An IHC assay was used to detect the expression levels of LETM1 in the tissues. The paraffin-embedded tissue samples were dewaxed and incubated with 3\% hydrogen peroxide for $30 \mathrm{~min}$ to inhibit endogenous peroxidase activity. Then the sections were infiltrated in citrate buffer and heated in a microwave for $10 \mathrm{~min}$ to carry out antigen retrieval. Subsequently the sections were incubated with the primary antibody anti-LETM1 (1:100, sc-271234; Santa Cruz Biotechnology, Inc., Santa Cruz, CA, USA) overnight at $4^{\circ} \mathrm{C}$. Then the sections were washed with phosphate-buffered saline (PBS) and the peroxidase-labeled goat anti-mouse secondary antibody was applied at room temperature for $1 \mathrm{~h}$. Next, the slides were stained with 3,3'-diaminobenzidine tetrahydrochloride (DAB) and hematoxylin for visualization. The intensity of LETM1 staining was scored as: 0 , none; 1 , weak; and 2 , strong. The proportion of positive tumor cells was recorded as follows: $1,1-25 \% ; 2,26-50 \% ; 3,51-75 \%$; and $4,76-100 \%$. The scores were multiplied to obtain a final score and the total expression of LETM1 was determined as either negative/weak expression (score $<4$ ) or overexpression (score $\geq 4$ ).

Cell transfection. The small interfering RNA (siRNA) targeting human LETM1 (si-LETM1) and the corresponding negative control (si-NC) were purchased from GenePharma (Shanghai, China). The sequence of LETM1 siRNA was: 5'-CCACAGAAUCGUGUCUGGAUCCACA-3'. When the cells were grown to $50 \%$, the aforementioned molecular products were transfected into bladder cancer cells using Lipofectamine 2000 (Invitrogen) according to the manufacturer's instructions. The medium containing the transfection reagents was removed $6 \mathrm{~h}$ after transfection, and $48 \mathrm{~h}$ later, the cells were harvested for the following assays.

Cell proliferation assay. Cell proliferation was assessed using Cell Counting Kit-8 (CCK-8; Dojindo Molecular Technologies Inc., Kumamoto, Japan). Briefly, $100 \mu 1$ of transfected cells were seeded into 96 -well plates at a density of 1,000 cells/well, and then $10 \mu \mathrm{l}$ of CCK-8 was added into each well at indicated time-points $(24,48,72$ and $96 \mathrm{~h})$ and incubation followed for $2 \mathrm{~h}$ at $37^{\circ} \mathrm{C}$. The absorbance at $450 \mathrm{~nm}$ was detected on a microplate spectrophotometer (BioTek Instruments Inc., Winooski, VT, USA).

Transwell migration and invasion assays. Transwell chambers (BD Biosciences, San Jose, CA, USA) were used for cell migration and invasion assays. For the invasion assay, each chamber was precoated with $25 \mu 1$ of Matrigel (BD Biosciences) at $37^{\circ} \mathrm{C}$ for $2 \mathrm{~h}$, whereas the chambers used for the migration assay were not precoated with Matrigel. Transfected cells $\left(5 \times 10^{4}\right)$ in $200 \mu \mathrm{l}$ serum-free medium were plated in the upper well of the chamber, and $600 \mu \mathrm{l}$ of RPMI-1640 medium containing $10 \%$ FBS was added into the lower chambers as a chemoattractant. After incubation at $37^{\circ} \mathrm{C}$ for $14 \mathrm{~h}$, the non-invading cells were removed with a cotton tip, and the cells that had migrated to the lower surface of the chamber were fixed with 95\% ethanol for $20 \mathrm{~min}$, stained with $0.1 \%$ crystal violet solution for $10 \mathrm{~min}$, washed for 3 times, air dried, photographed and counted in 5 randomly selected fields for each well using a light microscope (Olympus, Tokyo, Japan).

Cell cycle assay. For the cell cycle analysis, the transfected cells were cultured for $48 \mathrm{~h}$, digested with trypsin, washed with PBS, centrifuged and fixed in $75 \%$ ethanol at $4^{\circ} \mathrm{C}$ overnight. Then the cells were washed with PBS, centrifuged and resuspended in $1 \mathrm{ml}$ of PBS containing $1 \mathrm{mg} / \mathrm{ml}$ RNase A and $50 \mu \mathrm{g} / \mathrm{ml}$ propidium iodide. The cells were then incubated for $30 \mathrm{~min}$ at room temperature in the dark and analyzed immediately using a flow cytometer (BD Biosciences).

Western blot analysis. Ice-cold RIPA buffer (Sigma-Aldrich) containing a protease inhibitor was used to isolate protein from cells or tissues. A BCA protein assay kit was used to determine the concentration of total cellular protein according to the manufacturer's instructions. Then equal amounts of protein were loaded onto $10 \%$ SDS-PAGE and were transferred onto nitrocellulose membranes. The membranes were blocked in 5\% non-fat milk for $1 \mathrm{~h}$ and then incubated with the following primary antibodies: mouse anti-LETM1 (sc-271234) and mouse anti- $\beta$-actin (sc-130300) (both from Santa Cruz Biotechnology, Inc.), rabbit anti- $\beta$-catenin (no. 8480; Cell Signaling Technology, Danvers, MA, USA), rabbit anti-cyclin D1 (ab134175; Abcam, Cambridge, UK), and rabbit anti-c-Myc (no. 5605; Cell Signaling Technology), overnight at $4^{\circ} \mathrm{C}$. After being washed with PBST three times, the membranes were incubated with the corresponding secondary antibodies at room temperature for $1 \mathrm{~h}$. The protein bands were visualized using the Odyssey scanner (LI-COR Biosciences, Lincoln, NE, USA).

Statistical analysis. SPSS 16.0 software (SPSS Inc., Chicago, IL, USA) was used for statistical analysis. All the data are presented 
A

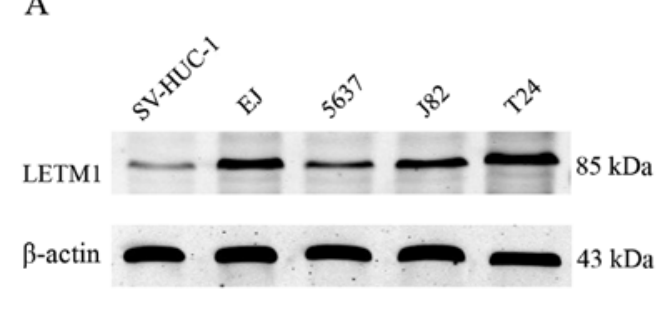

B

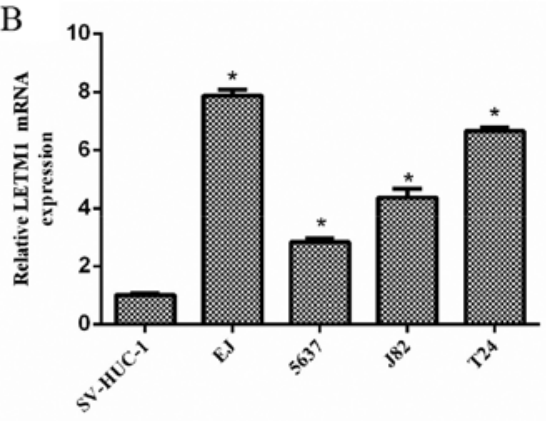

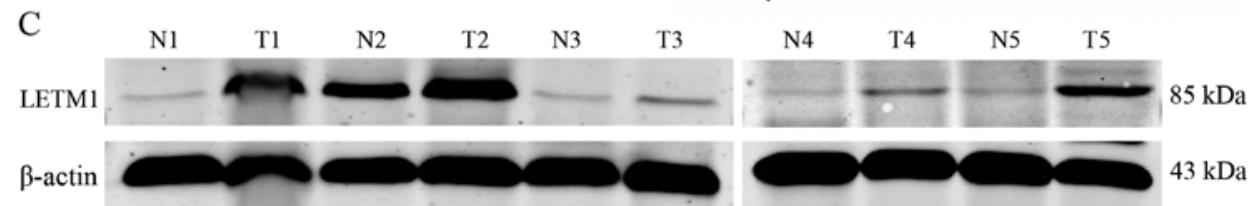

Figure 1. LETM1 is highly expressed in bladder cancer cell lines. (A and B) The protein and the mRNA expression of LETM1 was determined in four human bladder cancer cell lines (EJ, 5637, J82 and T24) and one normal human bladder transitional cell line SV-HUC-1 by western blot analysis and qRT-PCR, respectively. (C) The expression of LETM1 was determined in tumor tissues and their matched adjacent normal tissues by western blot analysis. $\beta$-actin was used as a loading control and the LETM1 mRNA level was normalized to the $\beta$-actin mRNA level. "p<0.05 vs. SV-HUC-1. LETM1, leucine zipper-EF-hand containing transmembrane protein 1.

as the means \pm standard deviation (SD) from at least three independent experiments. The immunohistochemical staining results were evaluated using Pearson's Chi-square test and the other experimental results were calculated using Student's t-test. A P-value $<0.05$ was considered to indicate a statistically significant result. The diagrams were drawn using GraphPad Prism 5 software (GraphPad Software, Inc., La Jolla, CA, USA).

\section{Results}

LETM1 is overexpressed in human bladder cancer cell lines and tissues. To explore the expression differences of LETM1 between the bladder cancer cells and the normal human bladder transitional cell line SV-HUC-1, western blotting and qRT-PCR were performed. As shown in Fig. 1A, the protein level of LETM1 was significantly higher in the bladder cancer cell lines, compared to that in the normal human bladder transitional cells. Moreover, LETM1 mRNA expression was further confirmed to be abundant in bladder cancer cell lines (Fig. 1B), which was consistent with the western blot analysis. Furthermore, we detected the protein expression of LETM1 in 5 pairs of tumor tissues and matched adjacent normal tissues by western blot analysis. As shown in Fig. 1C, the protein expression of LETM1 was higher in the bladder cancer tissues than that in the adjacent normal tissues.

To explore the correlation between the expression of LETM1 and the clinicopathological parameters of bladder cancer, immunohistochemistry was applied to examine the LETM1 protein expression level in 86 cases of bladder cancer tissues and 5 adjacent normal tissues. We found that negative LETM1 staining was observed in normal bladder tissues, and positive LETM1 staining was observed in bladder cancer tissues (Fig. 2). Moreover the expression of LETM1 in bladder cancer was associated with the pathological stage, lymph node metastasis and recurrence of bladder cancer $(p<0.05)$ (Table I). These results indicated that LETM1 was upregulated in bladder cancer cell lines and tissues, therefore we assumed that LETM1 may act as an oncogene in bladder cancer.
Table I. Relationship between the expression of LETM1 and the clinicopathological parameters in bladder cancer patients.

\begin{tabular}{lcccc}
\hline Variables & $\begin{array}{c}\text { No. of } \\
\text { cases }\end{array}$ & $\begin{array}{c}\text { LETM1 } \\
\text { overexpression } \\
\text { cases (\%) }\end{array}$ & $\chi^{2}$ & P-value $^{\mathrm{a}}$ \\
\hline Sex & & & 0.167 & 0.683 \\
$\quad$ Male & 57 & $36(63.2)$ & & \\
Female & 29 & $17(58.6)$ & & \\
Age (years) & & & 3.230 & 0.072 \\
$\quad 60$ & 39 & $20(51.3)$ & & \\
$\geq 60$ & 47 & $33(70.2)$ & & \\
Pathological & & & 9.466 & 0.009 \\
stage & & & & \\
Ta & 19 & $6(31.6)$ & & \\
T1 & 41 & $28(68.3)$ & & \\
$\geq$ T2 & 26 & $19(73.1)$ & & \\
Grade & & & 0.614 & 0.433 \\
Low & 27 & $15(55.6)$ & & \\
High & 59 & $38(64.4)$ & & \\
Lymph node & & & 10.185 & 0.001 \\
metastasis & & & & \\
No & 58 & $29(50.0)$ & & \\
Yes & 28 & $24(85.7)$ & & \\
Recurrence & & & 5.029 & 0.025 \\
No & 39 & $19(53.8)$ & & \\
Yes & 47 & $34(68.1)$ & & \\
\hline
\end{tabular}

${ }^{a}$ Using Pearson's Chi-square test. LETM1, leucine zipper-EF-hand containing transmembrane protein 1 .

Knockdown of LETM1 inhibits bladder cancer cell proliferation. To further explore the role of LETM1 in bladder cancer 

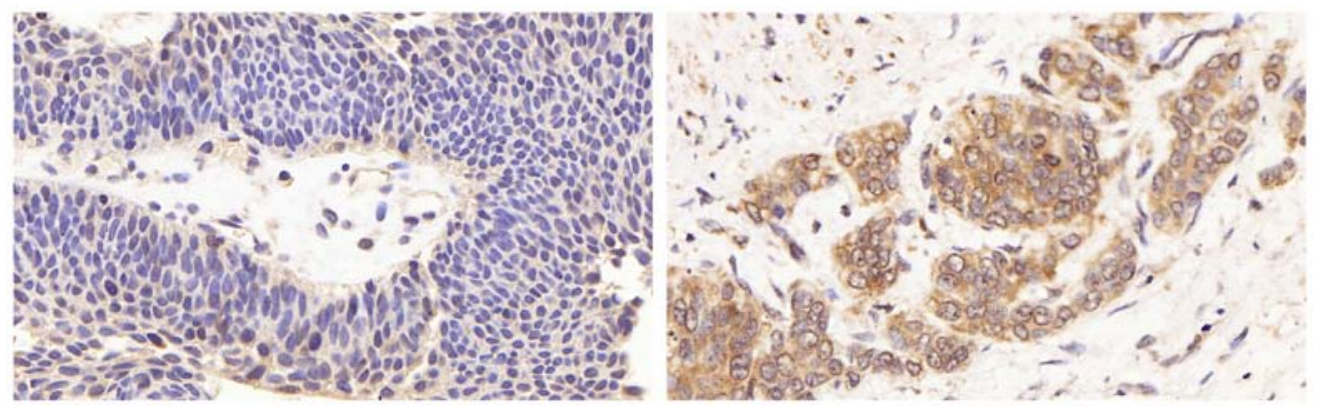

Figure 2. Determination of the expression of LETM1 by immunohistochemical staining. Negative LETM1 staining in the majority of normal bladder tissue (left) and positive LETM1 staining in the bladder cancer tissue (right). LETM1, leucine zipper-EF-hand containing transmembrane protein 1.

A
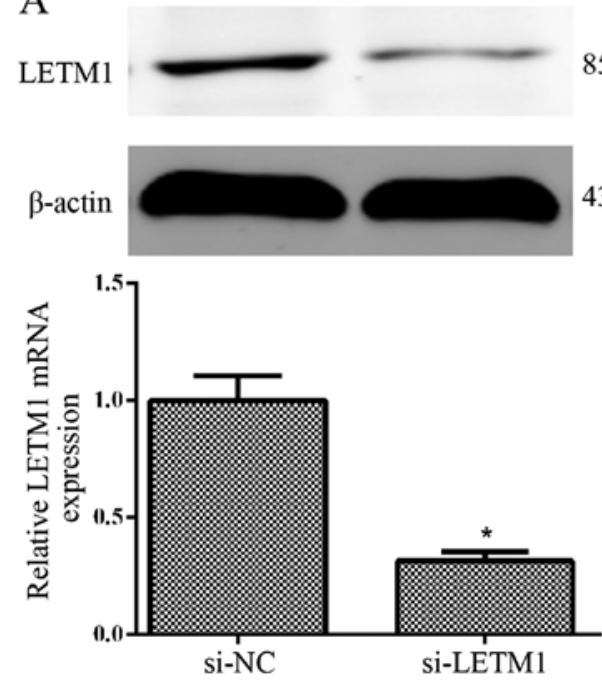

B

$85 \mathrm{kDa}$

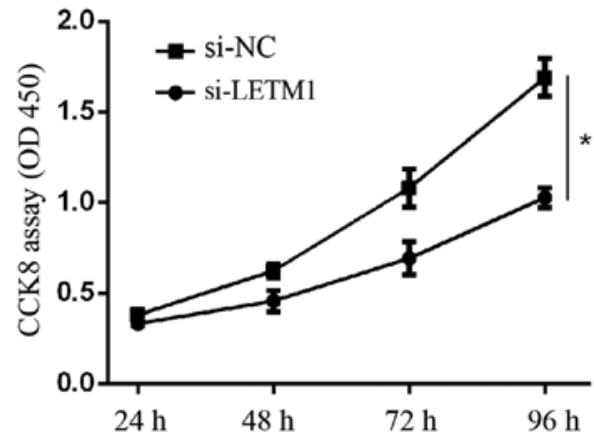

Figure 3. Knockdown of LETM1 inhibits cell proliferation in human bladder cancer cells. (A) The knockdown of LETM1 was confirmed in T24 cells by western blot analysis and qRT-PCR at 48 and $72 \mathrm{~h}$ after transfection, respectively. $\beta$-actin was used as a loading control and the LETM1 mRNA level was normalized to the $\beta$-actin mRNA level. (B) Cell proliferation was determined in the T24 cells transfected with si-LETM1 or si-NC using a CCK8 assay. * $\mathrm{p}<0.05$ vs. si-NC. LETM1, leucine zipper-EF-hand containing transmembrane protein 1.
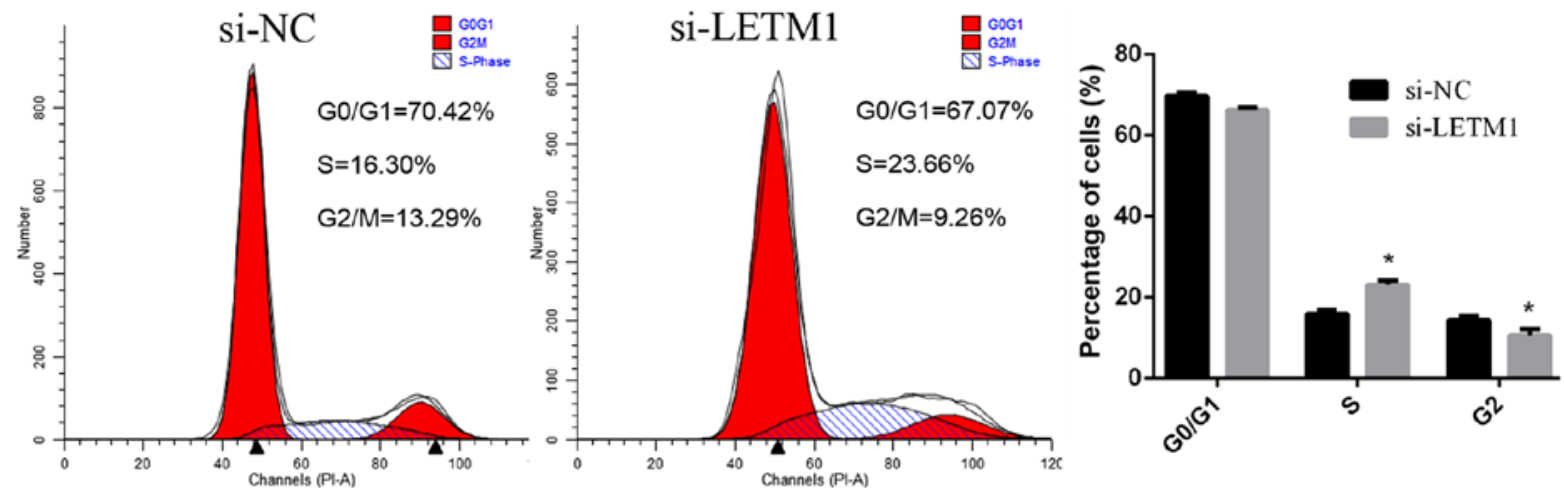

Figure 4. LETM1 knockdown induces accumulation of S-phase cells. Cell cycle distribution of T24 cells transfected with si-LETM1 or si-NC was determined using flow cytometric assay. " $\mathrm{p}<0.05$ vs. si-NC. LETM1, leucine zipper-EF-hand containing transmembrane protein 1.

cells, T24 cells were transfected with si-LETM1 or si-NC, and then the expression of LETM1 at the mRNA and protein level was detected at 48 and $72 \mathrm{~h}$ after transfection, respectively. We found that knockdown of LETM1 by si-LETM1 significantly decreased the expression of LETM1 at the mRNA and protein level in T24 cells (Fig. 3A). A CCK-8 proliferation assay revealed that knockdown of LETM1 markedly decreased the proliferation of T24 cells, when compared with the respective control (Fig. 3B). Collectively, knockdown of LETM1 markedly inhibited bladder cancer cell proliferation.

LETM1 knockdown induces accumulation of S-phase cells. To further investigate the possible mechanism underlying the cell growth inhibition effect by suppression of the expression 

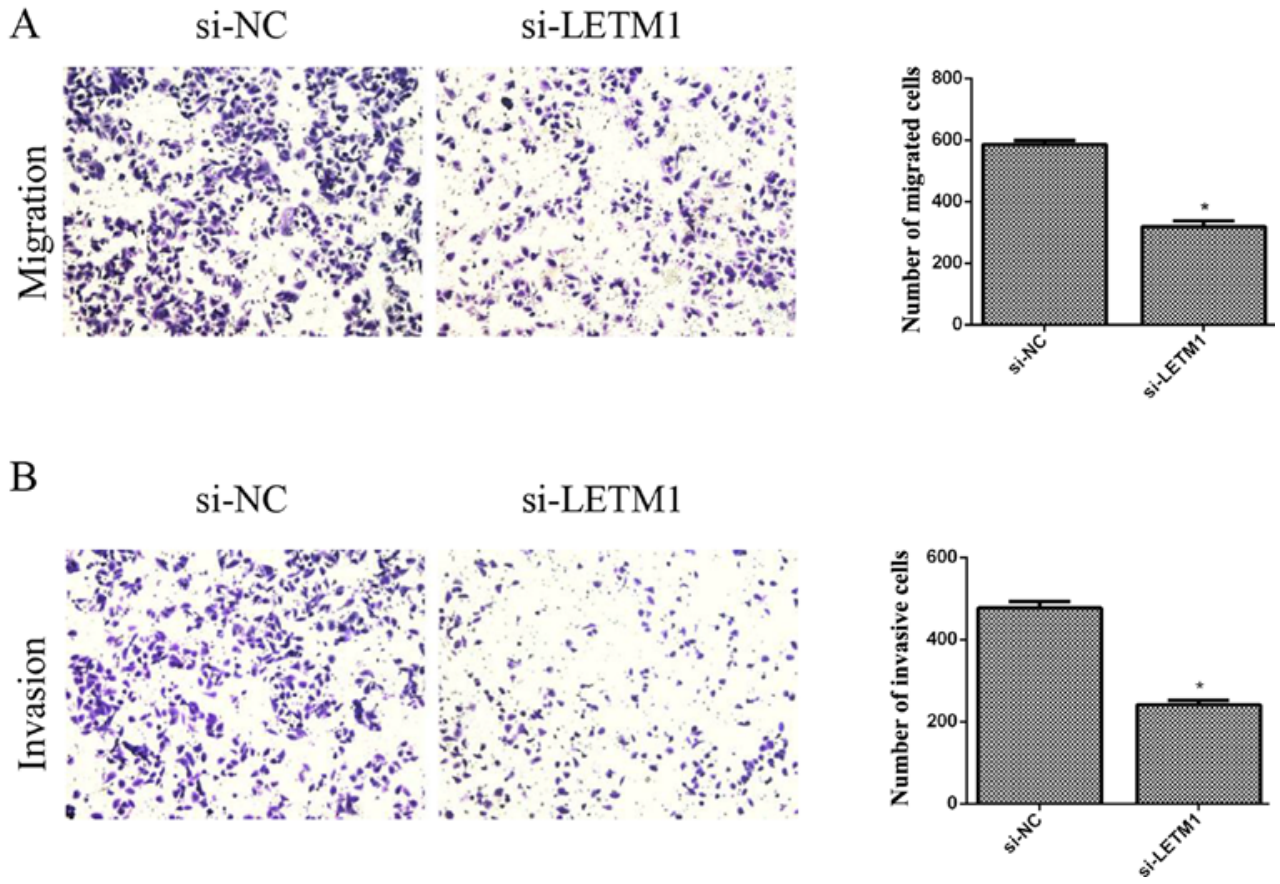

Figure 5. Knockdown of LETM1 inhibits cell migration and invasion in human bladder cancer cells. (A) The migration of T24 cells transfected with si-LETM1 or si-NC was evaluated by Transwell assay. (B) The invasion of T24 cells transfected with si-LETM1 or si-NC was evaluated by Transwell assay with Matrigel. * $\mathrm{p}<0.05$ vs. si-NC. LETM1, leucine zipper-EF-hand containing transmembrane protein 1.
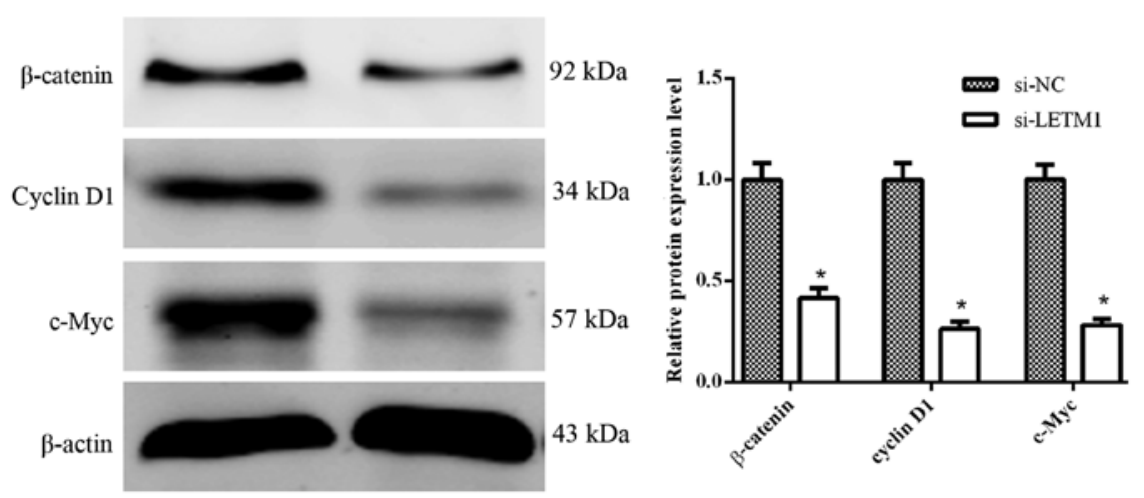

Figure 6. The silencing of LETM1 inhibits the activation of the Wnt/ $\beta$-catenin pathway in bladder cancer cells. The protein expression of $\beta$-catenin, cyclin D1 and c-Myc was analyzed using western blotting in T24 cells transfected with si-LETM1 or si-NC. $\beta$-actin was used as a loading control. "p $<0.05$ vs. si-NC. LETM1, leucine zipper-EF-hand containing transmembrane protein 1.

of LETM1 in bladder cancer cells, the cell cycle was analyzed using a flow cytometer. The percentage of cells at different phases revealed that LETM1 knockdown induced bladder cancer cell accumulation at the S phase (Fig. 4).

Knockdown of LETM1 inhibits bladder cancer cell migration and invasion. We then investigated the effects of LETM1 on the cell migration of bladder cancer cells using Transwell chambers without Matrigel. The results revealed that knockdown of LETM1 markedly inhibited the migration of T24 cells, when compared with the respective control (Fig. 5A). In addition, we explored the role of LETM1 on cell invasion of bladder cancer cells using Transwell chambers with Matrigel. As revealed in Fig. 5B, knockdown of LETM1 significantly suppressed the number of T24 cells that invaded through the Matrigel, as compared with the respective control group.
Knockdown of LETM1 inhibits the activation of the Wnt/ $\beta$-catenin pathway. Some evidence has indicated that the Wnt/ $\beta$-catenin pathway plays a critical role in the tumor progression and metastasis of human bladder cancer cells $(11,12)$. To further explore the effects of si-LETM1 on the Wnt/ß-catenin signaling pathway in human bladder cancer cells, we determined the protein expression of $\beta$-catenin, cyclin D1 and c-Myc when knocking down LETM1. As illustrated in Fig. 6, knockdown of LETM1 significantly suppressed the protein expression of $\beta$-catenin, cyclin D1 and c-Myc in T24 cells.

\section{Discussion}

Emerging evidence has indicated that many oncogene and anti-oncogene alterations are involved in the tumorigenesis of bladder cancer (13). Although great progress in diagnosis and treatment has been achieved in the past decades, the underlying 
molecular mechanism of bladder tumorigenesis still remains to be elucidated and targets for gene therapy are limited. In the present study, we demonstrated that the suppression of LETM1 significantly suppressed cell proliferation, migration and invasion and disrupted the cell cycle distribution.

Recent studies revealed that LETM1 is highly expressed in many types of human cancer and predicts poor prognosis $(8-10,14)$. This is consistent with our results which revealed that the expression of LETM1 was increased in bladder cancer and that upregulated LETM1 expression was related with pathological stage, lymph node metastasis and recurrence of bladder cancer. As for the molecular function of LETM1, Doonan et al found that knockdown of LETM1 caused accumulation of S-phase cells and the re-expression of LETM1 could reverse S-phase accumulation (15), which was consistent with our results, indicating that the suppression of LETM1 inhibited cell proliferation possibly by disrupting the cell cycle distribution. Piao et al found that the overexpression of LETM1 could induce necrotic cell death in HeLa cells by decreasing mitochondrial biogenesis and ATP production (8). However, Dimmer et al found that downregulation of LETM1 also caused necrotic cell death (16). It is still unclear how both the gain and loss of LETM1 causes similar phenotypes in cells.

The Wnt/ $\beta$-catenin pathway is an important signaling pathway involved in the malignant progression of various tumors, and regulates the proliferation, migration and invasion of cancer cells (17-19). When Wnt is activated, $\beta$-catenin translocates from the cytoplasm to the nucleus and stimulates proto-oncogene cyclin D1 and c-Myc transcription $(20,21)$. Cyclin D1 promotes cell proliferation by regulating the G1 phase progression of the cell cycle (22) and c-Myc encodes a transcription factor, that triggers selective gene expression amplification and promotes cell proliferation by genetic and epigenetic elimination of checkpoints (23). In our study, we found that knockdown of LETM1 inhibited the expression of $\beta$-catenin, cyclin D1 and $\mathrm{c}-\mathrm{Myc}$ in bladder cancer cells. These results indicated that silencing of LETM1-inhibited cell proliferation may partially be epigenetically inhibiting $\beta$-catenin, cyclin D1 and c-Myc expression. However, more research is warranted in order to explore the underlying mechanism of how LETM1 affects the Wnt/ $\beta$-catenin pathway. The expression of some specific indicators of Wnt/ $/$-catenin signaling and the nuclear localization of $\beta$-catenin need to be analyzed after knockdown of LETM1.

Collectively, our results revealed that the knockdown of LETM1 suppresses proliferation, migration and invasion of bladder cancer cells possibly via the suppression of the Wnt/ $\beta$-catenin signaling pathway. Therefore, our results indicate that LETM1 may be a novel target for bladder cancer therapy.

\section{Acknowledgements}

This study was supported by a research grant from the National Natural Science Foundation of China (no. 81370699).

\section{References}

1. Siegel RL, Miller KD and Jemal A: Cancer statistics, 2015. CA Cancer J Clin 65: 5-29, 2015.

2. Babjuk M, Böhle A, Burger M, Capoun O, Cohen D, Compérat EM, Hernández V, Kaasinen E, Palou J, Rouprêt M, et al: EAU guidelines on non-muscle-invasive urothelial carcinoma of the bladder: Update 2016. Eur Urol 3: 447-461, 2017.
3. Kamat AM,Hahn NM, Efstathiou JA, Lerner SP, Malmström PU, Choi W, Guo CC, Lotan Y and Kassouf W: Bladder cancer. Lancet 388: 2796-2810, 2016.

4. Chester JD, Hall GD, Forster M and Protheroe AS: Systemic chemotherapy for patients with bladder cancer - current controversies and future directions. Cancer Treat Rev 30: 343-358, 2004.

5. Budman LI, Kassouf W and Steinberg JR: Biomarkers for detection and surveillance of bladder cancer. Can Urol Assoc J 2: 212-221, 2008.

6. Hart L, Rauch A, Carr AM, Vermeesch JR and O'Driscoll M: LETM1 haploinsufficiency causes mitochondrial defects in cells from humans with Wolf-Hirschhorn syndrome: Implications for dissecting the underlying pathomechanisms in this condition. Dis Model Mech 7: 535-545, 2014.

7. Endele S, Fuhry M, Pak SJ, Zabel BU and Winterpacht A: LETM1, a novel gene encoding a putative EF-hand $\mathrm{Ca} 2^{+-}$-binding protein, flanks the Wolf-Hirschhorn syndrome (WHS) critical region and is deleted in most WHS patients. Genomics 60: 218-225, 1999.

8. Piao L, Li Y, Kim SJ, Byun HS, Huang SM, Hwang SK, Yang KJ, Park KA, Won M,Hong J, et al: Association of LETM1 and MRPL36 contributes to the regulation of mitochondrial ATP production and necrotic cell death. Cancer Res 69: 3397-3404, 2009.

9. Chen L, Yang Y, Liu S, Piao L, Zhang Y, Lin Z and Li Z: High expression of leucine zipper-EF-hand containing transmembrane protein 1 predicts poor prognosis in head and neck squamous cell carcinoma. Biomed Res Int 2014: 850316, 2014.

10. Wang CA, Liu Q, Chen Y, Liu S, Xu J, Cui X, Zhang Y and Piao L: Clinical implication of leucine zipper/EF hand-containing transmembrane-1 overexpression in the prognosis of triple-negative breast cancer. Exp Mol Pathol 98: 254-259, 2015.

11. Ahmad I: The role of WNT signalling in urothelial cell carcinoma. Ann R Coll Surg Eng1 97: 481-486, 2015.

12. Pierzynski JA, Hildebrandt MA, Kamat AM, Lin J, Ye Y, Dinney CP and Wu X: Genetic variants in the Wnt $/ \beta$-catenin signaling pathway as indicators of bladder cancer risk. J Urol 194: 1771-1776, 2015.

13. Costello JC and Theodorescu D: Decade in review-bladder cancer: International progress: From cytology to genomics. Nat Rev Urol 11: 609-610, 2014.

14. Li N, Zheng Y, Xuan C, Lin Z, Piao L and Liu S: LETM1 overexpression is correlated with the clinical features and survival outcome of breast cancer. Int J Clin Exp Pathol 8: 12893-12900, 2015.

15. Doonan PJ, Chandramoorthy HC, Hoffman NE, Zhang X, Cárdenas C, Shanmughapriya S, Rajan S, Vallem S, Chen X, Foskett JK, et al: LETM1-dependent mitochondrial $\mathrm{Ca}^{2+}$ flux modulates cellular bioenergetics and proliferation. FASEB J 28: 4936-4949, 2014.

16. Dimmer KS, Navoni F, Casarin A, Trevisson E, Endele S, Winterpacht A, Salviati L and Scorrano L: LETM1, deleted in Wolf-Hirschhorn syndrome is required for normal mitochondrial morphology and cellular viability. Hum Mol Genet 17: 201-214, 2008.

17. Wang HS, Nie X, Wu RB, Yuan HW, Ma YH, Liu XL, Zhang JY, Deng XL, Na Q, Jin HY, et al: Downregulation of human Wnt3 in gastric cancer suppresses cell proliferation and induces apoptosis. Onco Targets Ther 9: 3849-3860, 2016.

18. Li K, Zhou ZY, Ji PP and Luo HS: Knockdown of $\beta$-catenin by siRNA influences proliferation, apoptosis and invasion of the colon cancer cell line SW480. Oncol Lett 11: 3896-3900, 2016.

19. Wu D, Li L and Yan W: Knockdown of TC-1 enhances radiosensitivity of non-small cell lung cancer via the Wnt/ $\beta$-catenin pathway. Biol Open 5: 492-498, 2016.

20. Klein EA and Assoian RK: Transcriptional regulation of the cyclin D1 gene at a glance. J Cell Sci 121: 3853-3857, 2008.

21. Qin X, Zhang H, Zhou X, Wang C, Zhang H, Zhang X and Ye L: Proliferation and migration mediated by Dkk-1/Wnt/ $\beta$-catenin cascade in a model of hepatocellular carcinoma cells. Transl Res 150: 281-294, 2007.

22. Cicatiello L, Addeo R, Sasso A, Altucci L, Petrizzi VB, Borgo R, Cancemi M, Caporali S, Caristi S, Scafoglio C, et al: Estrogens and progesterone promote persistent CCND1 gene activation during G1 by inducing transcriptional derepression via c-Jun/c-Fos/ estrogen receptor (progesterone receptor) complex assembly to a distal regulatory element and recruitment of cyclin D1 to its own gene promoter. Mol Cell Biol 24: 7260-7274, 2004.

23. Stine ZE, Walton ZE, Altman BJ, Hsieh AL and Dang CV: MYC, metabolism and cancer. Cancer Discov 5: 1024-1039, 2015. 